\title{
Strong Phonon-Plasmon Coupling Between Nanoscale Antennas
}

\author{
Maureen Lagos ${ }^{1}$, Philip Batson ${ }^{2}$, Ulrich Hohenester ${ }^{3}$ and Gianluigi Botton ${ }^{4}$ \\ ${ }^{1}$ McMaster University, Hamilton, Ontario, Canada, ${ }^{2}$ Rutgers University, Piscataway, New Jersey, United \\ States, ${ }^{3}$ University of Graz, Graz, Steiermark, Austria, ${ }^{4}$ McMaster University \& Canadian Centre for \\ Electron Microscopy \& Canadian Light Source Inc, Hamilton, Ontario, Canada
}

Over the last few years, the development of highly monochromatic atom-wide electron beams have given us opportunities to explore collective excitations (plasmon, phonons) and map their corresponding inelastic scattering in nanoscale materials with high spatial resolution. For instance, near-infrared plasmons in metal structures of a few micrometers in length were probed by fast electrons, unveiling exotic properties of plasmonic wires [1]. More recently, bulk and surface phonons were probed in nanostructures with nanometric spatial resolution [2], and it was shown that inelastic scattering by surface phonon polaritons has a delocalized nature [3], like their surface plasmonic counterparts. In this work, we present an experimental and theoretical EELS study of the interaction between plasmon and phonon polaritons sustained in two interacting nanoscale antennas (two-antenna system). In particular, we focus on measuring the dispersion of hybrid phonon-plasmon modes and on the scattering delocalization across the antenna interfaces.

We have used a Nion UltraStem microscope equipped with a monochromator to study the phonon and infrared plasmon response using a $\sim 1.5$ - 2 A probe with an energy spread of $\sim 9 \mathrm{meV}$, at $60 \mathrm{kV}[4]$. We have fabricated rod-like $\mathrm{SiO}_{2}$ phononic antennas and single rod-like Al plasmonic antennas of different lengths, in order to evaluate their response in the (near-)infrared range. We have also fabricated twoantenna systems, with each rod-like component consisting of one plasmonic and one phononic antenna, which were attached together (see schematic in Fig. 1). The antenna lengths $(L)$ were ranging between 1 and $6 \mu \mathrm{m}$. Our spatially-resolved EELS studies were performed along/across the antennas, aiming to study the spatial distribution of the polaritonic excitations.

We have initially probed $\mathrm{SiO}_{2}$ antennas of different lengths and found that the surface phononic response is confined within the Reststrahlen bands of the material, as expected in systems sustaining surface phonons modes [5]. By changing the length of the $\mathrm{SiO}_{2}$ antenna, we have found that the surface scattering remains constrained within the Reststrahlen bands, which indicates less flexibility for tuning their phononic response. In contrast, for Al plasmonic antennas, we can tune their plasmonic response over a wider range by adjusting their length. For instance, the dipolar modes of single aluminum antennas of 1 and $6 \mu \mathrm{m}$ in length have energies at $\sim 460 \mathrm{meV}$ and $\sim 75 \mathrm{meV}$, respectively. This clearly shows plasmonic tunability over a wider spectral range $(\Delta \mathrm{E} \sim 540 \mathrm{meV})$, in comparison to the phononic antenna $(\Delta \mathrm{E} \sim 10$ - $40 \mathrm{meV}$ ). Higher-order plasmon modes were also detected at higher energies depending on the Al antenna length [1].

To study the interaction between surface plasmon and phonon polaritons, we have put the antennas together. The plasmonic response of the aluminum structure was tuned by adjusting its length in such a way that its response lies within the upper Reststrahlen band of the silica structure. This situation was achieved with structures of about $3 \mu \mathrm{m}$ in length. The corresponding EELS spectra revealed a resonance split of about $20 \mathrm{meV}$, as a result of the strong coupling of those two excitations (See two EELS spectra in Inset of Fig.1). Structures of slightly different lengths $(2.7,3.0,3.1,3.4 \mu \mathrm{m})$ were also prepared to study 
closely the split response within the band (indicated by blue dots in Fig. 1). The split is a very robust phenomenon which reveals the formation of a new hybrid phonon-plasmon excitation, due to the interaction between the dipolar modes of each antenna, one due to the collective motions of electrons (plasmon) in the aluminum and other due to the collective motion of ions (phonons) in the silica.

In addition, we have measured the relationship between the oscillation frequency and the wave-vector of the hybrid mode. The corresponding dispersion plot is shown in Fig. 1. Note that a split in the dispersion curve shows up within the upper Reststrahlen band (gray band in the plot). Our calculations, based on dielectric theory, also reveal frequency splits in the two Reststrahlen bands of the silica, an indication of strong coupling. The linear dispersive character indicates that this hybrid excitation possesses a photonlike nature and it propagates along the structure with a velocity smaller than the speed of light. This reduction might be induced by the structural features on the surface which limits its propagation. We have also mapped the scattering associated with the hybrid excitation at 132 and $152 \mathrm{meV}$ and found that its spatial distribution is similar to the distribution of plasmonic dipolar modes in rod-like structures [1]. Furthermore, we have conducted EELS measurements across the $\mathrm{SiO}_{2} / \mathrm{Al}$ interfaces and found that the scattering signal is strongly dependent on the probe position. We have obtained strong suppression of the $152 \mathrm{meV}$ resonances in the silica side and a reduction of the energy split, probably due to bulk contributions at around $145 \mathrm{meV}$.

In summary, we have probed a two-antenna system composed by one plasmonic rod-like Al structure attached to a phononic rod-like $\mathrm{SiO}_{2}$ structure. We have shown that the polaritonic response can be tuned by adjusting the length of the bi-layer antenna structure and found evidence of strong coupling between plasmon and phonons, as revealed by the energy split, extending our understanding of phonon and plasmon polariton excitations in finite-size objects [6].

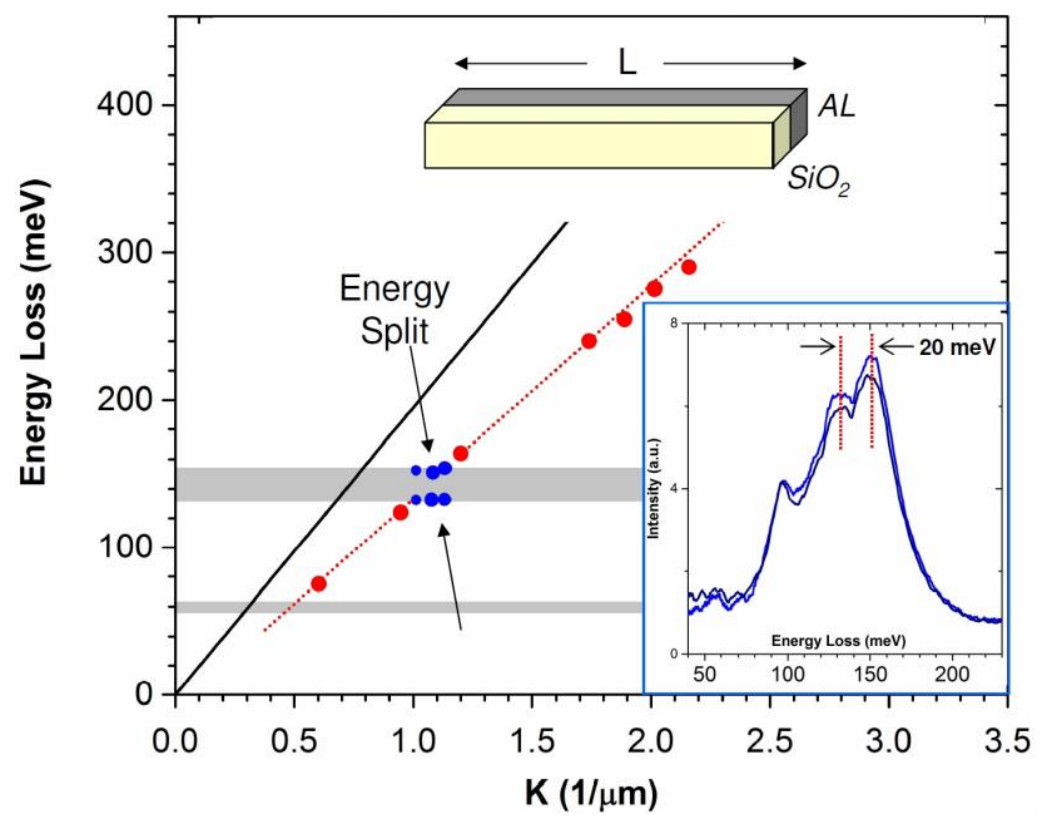

Figure 1. Dispersion plot of a hybrid excitation generated by two adjacent plasmonic and phononic antennas of length L, as indicated in the schematic (top part). Note the energy split formed in the upper Reststrahlen band (upper gray band). Experimental data was highlighted in blue inside the band, while data outside is shown by red dots. The black lines represent the light and line and the dotted line was 
plotted to guide the eyes. Inset shows EELS spectra acquired for the two-antenna systems revealing a resonant split $(20 \mathrm{meV})$, an indication of strong coupling between surface phonon and plasmon polaritons.

\section{References}

[1] D. Rossouw, G. A. Botton, Physical Review Letters 110 (2013) 066801.

[2] M. J. Lagos, et al, Nature 543 (2017) 529.

[3] U. Hohenester, et al, Physical Review B 97 (2018) 165418.

[4] O. Krivanek, et al, Nature 514 (2014) 209.

[5] M. J. Lagos, et al, Microscopy 67 (2018) i3.

[6] For financial support, M.J.L. and G.A.B. acknowledge the Natural Sciences and Engineering Research Council (NSERC) under a discovery grant; P.E.B. acknowledges support of U.S. Department of Energy, Office of Science, Basic Energy Sciences, under award no. DE-SC0005132. The authors also acknowledge L. C. Feldman, V. Amarasinghe and A. Knigths for providing samples, and A. Trugler and H. Yang for providing assistance with simulations and experiments. 